\title{
Achievable Rates for Two User Cooperative OFDMA
}

\author{
Sezi Bakım Onur Kaya \\ Department of Electronics Engineering \\ Iş1k University \\ Şile, Istanbul, Turkey \\ sezi.bakim@isik.edu.tr_onurkaya@isikun.edu.tr
}

\begin{abstract}
We propose two encoding strategies for a two user cooperative Orthogonal Frequency Division Multiple Access (OFDMA) system, based on block Markov superposition encoding. We obtain the expressions for the resulting achievable rate regions for both encoding strategies, and evaluate them through simulations. We show that, by allowing for re-partitioning and re-encoding of the cooperative messages across subchannels, it is possible to better exploit the diversity created by OFDMA, and higher rates can be achieved.
\end{abstract}

\section{INTRODUCTION}

OFDMA provides a relatively simple way of assigning available bandwidth to users, while avoiding multi-user interference; and at the same time, it converts a frequency selective fading channel, to parallel flat fading subchannels, hence reducing the effects of intersymbol interference. However, like all orthogonal multiple accessing techniques, OFDMA incurs a loss in achievable rates. Moreover, in wireless channels, what is considered as interference is in fact side information, and combined with the diversity created by the orthogonal subchannels in OFDMA, this side information can be carefully taken advantage of to increase the rates achievable by the users.

In OFDMA, one way of exploiting the diversity created across the orthogonal subchannels is resource (i.e., power, subchannel) allocation, which has been studied extensively in the literature. One example is [1], where the authors solve the power and subchannel allocation problem in two phases. In the first phase, each subchannel is assigned to the user with the best channel state; in the second, power allocation is executed by single user water-filling. A similar problem was solved in [2] by optimizing a utility function, which is assumed to be a function of the rates. There, Karush-Kuhn-Tucker (KKT) conditions were used and an algorithm with low complexity was proposed. The problems of downlink subchannel and power allocation and continuous and discrete rate adaptation were solved in [3], by using ergodic rates as the objective function, and Lagrangian techniques. These works, as well as many others, all assume orthogonal transmissions, and do not take into account the possibility of cooperation based on side information provided by the channel.

The idea of cooperative communications roots from the simplest form of cooperative channel model: the relay channel. This channel was first studied in [4]. Later, in the seminal paper [5], fundamental capacity and achievable rate theorems for the relay channel were proved, and several coding and decoding techniques were proposed. The extension of the one sided cooperative relay model to mutual cooperation was made possible by the introduction of multiple access channels (MAC) with generalized feedback [6], [7]. In [7], an achievable rate

This work was supported by The Scientific \& Technological Research Council of Turkey, Grant 108E208. region, which was larger than that of [6], was obtained by utilizing block Markov superposition encoding and backward decoding. More recently, in [8], the MAC with generalized feedback was used to model a fading cooperative additive white Gaussian noise (AWGN) channel, and the results therein made cooperative communications very attractive.

The use of cooperative protocols in OFDM systems was investigated extensively by many authors over the recent years. In [9], the authors obtained bounds on pairwise error probability for single antenna OFDM systems employing cooperative convolutional codes. In [10], per subcarrier hybrid cooperation strategies were proposed, with the goal of minimizing the error probability. Methods for subcarrier selection in multihop OFDM systems were developed in [11]. Resource allocation for an OFDMA system where each user is allowed to transmit and receive at the same time, but necessarily on different subcarriers, was considered in [12]. An amplify-and-forward (AF) scheme based on time-division duplex transmissions was employed in [13] with the aim of maximizing system throughput and enhancing fairness in a cooperative OFDMA uplink system.

The works in the literature related to cooperative OFDM, some examples of which are listed above, either consider a one sided cooperation strategy, or a mutually cooperative strategy based on two parallel dedicated relay channels, or mutual cooperation based on a time division protocol. In this paper, without imposing any prior constraints on which users will use which subchannels, we propose two full duplex cooperative encoding strategies: intra-subchannel and intersubchannel cooperative encoding. These strategies use decode and forward (DF) approach and are based on block Markov superposition encoding. Intra-subchannel cooperative encoding is an extension of the two user cooperative strategy in [7] and [8] to OFDMA, and inter-subchannel cooperative encoding is a novel method which allows for re-partitioning and reencoding of the cooperative messages across subchannels. We obtain the achievable rate regions for both strategies, and compare them with non-cooperative OFDMA capacity region. We demonstrate through simulations that, the new encoding strategies provide significant rate improvements.

\section{SySTEM MODEL}

We consider a two user cooperative OFDMA system with $N$ subchannels, modelled by,

$$
\begin{aligned}
& Y_{0}^{(i)}=\sqrt{h_{10}^{(i)}} X_{1}^{(i)}+\sqrt{h_{20}^{(i)}} X_{2}^{(i)}+Z_{0}^{(i)}, \\
& Y_{1}^{(i)}=\sqrt{h_{21}^{(i)}} X_{2}^{(i)}+Z_{1}^{(i)} \\
& Y_{2}^{(i)}=\sqrt{h_{12}^{(i)}} X_{1}^{(i)}+Z_{2}^{(i)},
\end{aligned}
$$


where, for each subchannel $i \in\{1, \ldots, N\}, X_{k}^{(i)}$ is the symbol transmitted by node $k, Z_{j}^{(i)}$ is the zero-mean additive white Gaussian noise at node $j, h_{k j}^{(i)}$ is the fading coefficient between nodes $k$ and $j$, and $Y_{j}^{(i)}$ is the symbol received at node $j$; with $k \in\{1,2\}$ and $j \in\{0,1,2\}$. Here, the receiver is denoted by $j=0$. The variance of $Z_{j}^{(i)}$ is given by $\sigma_{j}^{(i)}{ }^{2}$. To simplify the notation throughout the paper, we define the normalized fading coefficients $s_{k j}^{(i)}=\frac{h_{k j}^{(i)}}{\sigma_{j}^{(i)^{2}}}$.

Note that, this model can be extended to a more general setting with many users, if we assign different subsets of available subchannels to separate pairs of users, and the results we obtain in this paper will be readily extendable, thanks to the orthogonal nature of the channel. Clearly, in that scenario one also needs to address issues such as optimal grouping of cooperative partners, and assignment of subchannels to those pairs, which are interesting extensions to this work but are beyond the scope of this paper.

\section{Coding Techniques And RAte Regions for COOPERATIVE OFDMA}

The channel model given in (1)-(3) consists of $N$ orthogonal two user cooperative multiple access channels (CMAC) in parallel, over which users 1 and 2 communicate with a common receiver. Therefore, the encoding and decoding techniques known for the two user CMAC [8] can be easily extended to this system with $N$ subchannels in parallel, by simply dividing the total message to be transmitted into smaller submessages, and encoding each submessage independently over the orthogonal cooperative channels. This approach, which we call intra-subchannel cooperative encoding, is relatively easy to implement, but it does not allow us to take full advantage of the diversity created across subchannels by the OFDM system. For instance, if on one subchannel, the inter-user link is very strong, but user-destination links are consistently very weak, the total data rate of the submessage on that link will be dictated by the weaker link. If, however, we allow submessages received by a user on each subchannel to be combined, re-encoded and forwarded to the intended receiver by a re-allocation of the received message onto the subchannels, we will potentially obtain better rates. Therefore, we propose an implementation of such an approach, and call it inter-subchannel cooperative encoding. Since both of the encoding policies rely on the same message generation process, we first discuss how the messages to be transmitted are formed. Next, the two encoding policies briefly explained above, will be presented in more detail and their corresponding achievable rate regions will be derived.

\section{A. Message Generation}

We assume that users 1 and 2 have independent messages, $w_{1}$ and $w_{2}$ respectively, to be conveyed to the receiver. These messages are first divided into two submessages, i.e., $w_{1}=$ $\left\{w_{10}, w_{12}\right\}$ and $w_{2}=\left\{w_{20}, w_{21}\right\}$, as in [7], [8]. Here, $w_{k j}$ is intended to be decoded by user $j$ and the receiver, and $w_{k 0}$ is intended to be decoded by the receiver, where $j, k \in\{1,2\}$ and $j \neq k$.
These two submessages are further divided into $N$ submessages each, to be separately transmitted over $N$ subchannels:

$$
\begin{aligned}
& w_{k 0}=\left\{w_{k 0}^{(1)}, \ldots, w_{k 0}^{(N)}\right\}, \\
& w_{k j}=\left\{w_{k j}^{(1)}, \ldots, w_{k j}^{(N)}\right\} .
\end{aligned}
$$

The corresponding submessages over each subchannel have the rates $\left\{R_{k 0}^{(1)}, \ldots, R_{k 0}^{(N)}\right\}$ and $\left\{R_{k j}^{(1)}, \ldots, R_{k j}^{(N)}\right\}$, respectively.

\section{B. Intra-subchannel Cooperative Encoding}

This encoding strategy is a rather trivial extension of the superposition block Markov encoding used in scalar channels [7], [8], [14], to OFDMA. The encoding in each subchannel is done independently, by mapping the submessages $w_{k 0}^{(i)}$ and $w_{k j}^{(i)}$ onto codewords $X_{k}^{(i)}$ for each subchannel $i$. Following the notation in [8] and [14], and the message generation process described in (4), the transmitters divide their messages $w_{k}(b)$ in block $b=1, \cdots, B$, into submessages $\left\{w_{k 0}^{(i)}[b], w_{k j}^{(i)}[b]\right\}_{i=1}^{N}$. These submessages are then encoded using block Markov encoding, i.e.,

$$
\begin{aligned}
& X_{k 0}^{(i)}\left(w_{k 0}^{(i)}[b], w_{k j}^{(i)}[b-1], \hat{w}_{j k}^{(i)}[b-1]\right), \\
& X_{k j}^{(i)}\left(w_{k j}^{(i)}[b], w_{k j}^{(i)}[b-1], \hat{w}_{j k}^{(i)}[b-1]\right), \\
& U_{k}^{(i)}\left(w_{k j}^{(i)}[b-1], \hat{w}_{j k}^{(i)}[b-1]\right) .
\end{aligned}
$$

Here, $X_{k 0}^{(i)}$ carries the fresh information intended for the receiver, $X_{k j}^{(i)}$ carries the information intended for transmitter $j$ for cooperation in the next block and $U_{k}^{(i)}$ is the common information sent by both transmitters for the resolution of the remaining uncertainty from the previous block, all of which are transmitted over the $i^{\text {th }}$ subchannel and chosen from unit Gaussian distributions. The caret, as in $\hat{w}_{j k}^{(i)}[b-1]$, will be used to denote the estimates of messages throughout the paper. Finally, user $k$ 's codeword in subchannel $i$ is formed by the superposition of the codewords (5)-(7),

$$
X_{k}^{(i)}=\sqrt{p_{k 0}^{(i)}} X_{k 0}^{(i)}+\sqrt{p_{k j}^{(i)}} X_{k j}^{(i)}+\sqrt{p_{U_{k}}^{(i)}} U_{k}^{(i)},
$$

for $j, k \in\{1,2\}, k \neq j$. Note that, the codewords (5)-(7) in each subchannel, are assigned separate powers, which are required to satisfy the following average power constraints:

$$
\sum_{i} p_{k 0}^{(i)}+p_{k j}^{(i)}+p_{U_{k}}^{(i)}=\sum_{i} p_{k}^{(i)} \leq \bar{p}_{k}, \quad k=1,2 .
$$

Due to the orthogonal structure of OFDMA, the signals in separate subchannels may be independently decoded using backwards decoding at the receiver, without interfering with each other. Therefore, by suitably extending the results in [8] to $N$ parallel subchannels, it can be shown that the rate region given in the following theorem is achievable:

Theorem 1: For a two user cooperative OFDMA channel which employs intra-subchannel cooperative encoding described by (5)-(8), an achievable rate region is given by the closure of the convex hull of all rate pairs $\left(R_{1}, R_{2}\right)$ satisfying (10)(15), where $R_{1}=\sum_{i=1}^{N} R_{10}^{(i)}+R_{12}^{(i)}, R_{2}=\sum_{i=1}^{N} R_{20}^{(i)}+R_{21}^{(i)}$, and the convex hull is taken over all power allocation policies 
that satisfy (9).

$$
\begin{aligned}
R_{12}^{(i)}<C_{12}^{(i)} \triangleq E\left[\log \left(1+\frac{s_{12}^{(i)} p_{12}^{(i)}}{s_{12}^{(i)} p_{10}^{(i)}+1}\right)\right], \\
R_{21}^{(i)}<C_{21}^{(i)} \triangleq E\left[\log \left(1+\frac{s_{21}^{(i)} p_{21}^{(i)}}{s_{21}^{(i)} p_{20}^{(i)}+1}\right)\right], \\
R_{10}^{(i)}<C_{10}^{(i)} \triangleq E\left[\log \left(1+s_{10}^{(i)} p_{10}^{(i)}\right)\right], \\
R_{20}^{(i)}<C_{20}^{(i)} \triangleq E\left[\log \left(1+s_{20}^{(i)} p_{20}^{(i)}\right)\right], \\
R_{10}^{(i)}+R_{20}^{(i)}<C_{0}^{(i)} \triangleq E\left[\log \left(1+s_{10}^{(i)} p_{10}^{(i)}+s_{20}^{(i)} p_{20}^{(i)}\right)\right], \\
R_{1}^{(i)}+R_{2}^{(i)}<C_{s}^{(i)} \triangleq E\left[\operatorname { l o g } \left(1+s_{10}^{(i)} p_{1}^{(i)}+s_{20}^{(i)} p_{2}^{(i)}\right.\right. \\
\left.\left.+2 \sqrt{s_{10}^{(i)} s_{20}^{(i)} p_{U_{1}}^{(i)} p_{U_{2}}^{(i)}}\right)\right] .
\end{aligned}
$$

It is more instructive to express the rate constraints in terms of $R_{1}$ and $R_{2}$ directly, rather than expressing them in terms of the rates of individual submessages as in Theorem 1. This is done in the following corollary.

Corollary 1: The achievable rate region given in Theorem 1 is equivalent to the closure of the convex hull of all rate pairs $\left(R_{1}, R_{2}\right)$ satisfying

$$
\begin{aligned}
R_{1} & <\sum_{i} \min \left\{C_{12}^{(i)}+C_{10}^{(i)}, C_{s}^{(i)}\right\}, \\
R_{2} & <\sum_{i} \min \left\{C_{21}^{(i)}+C_{20}^{(i)}, C_{s}^{(i)}\right\}, \\
R_{1}+R_{2} & <\sum_{i} \min \left\{C_{s}^{(i)}, C_{12}^{(i)}+C_{21}^{(i)}+C_{0}^{(i)}\right\} .
\end{aligned}
$$

The minimum operations, required over each subchannel as in (16)-(18), prevent us from efficiently exploiting the diversity offered by OFDMA. Therefore, we propose a new encoding strategy, explained in the following section, which removes the obligation to transmit the same submessages on user-user and user-receiver links over each subchannel, thereby moving the minimum operations in (16)-(18) outside of the summations.

\section{Inter-subchannel Cooperative Encoding}

The block Markov encoding strategy relies on users decoding part of each other's messages in each block, and re-encoding them in the next block. Although this can be done on a subchannel (and hence submessage) basis as described in Section III-B, it can also be done by re-encoding the overall message received over all subchannels, by an appropriate repartitioning of that message to subchannels, to be used in conjunction with the message generation process described in Section III-A.

Let us denote, the estimate at user $j$, of the message transmitted in the previous block by user $k$ over subchannel $i$, by $\hat{w}_{k j}^{(i)}$, $j \neq k$. Since user $j$ 's real objective is to decode the overall message $w_{k j}$ and re-encode it, in the re-encoding process $w_{k j}$ can be divided into new submessages with different rates:

$$
\begin{aligned}
& w_{12}=\left\{v_{12}^{(1)}, \ldots, v_{12}^{(N)}\right\} \\
& w_{21}=\left\{v_{21}^{(1)}, \ldots, v_{21}^{(N)}\right\} .
\end{aligned}
$$

The corresponding submessages over each subchannel have the rates $\left\{R_{12}^{\prime}(1), \ldots, R_{12}^{\prime(N)}\right\}$ and $\left\{R_{21}^{\prime(1)}, \ldots, R_{21}^{\prime(N)}\right\}$, respectively. It is assumed that a table to match the new cooperative submessages, $v_{k j}^{(i)}$, to the cooperative submessages received in the previous block, $w_{k j}^{(i)}$, is available at both of the users and the receiver, hence, the receiver can obtain $w_{k j}$ upon decoding $v_{k j}^{(i)}$ for all $i$.

It is important to note that, since $\left\{w_{k j}^{(i)}\right\}_{i=1}^{N}$ and $\left\{v_{k j}^{(i)}\right\}_{i=1}^{N}$, are just different partitionings of the same message $w_{k j}$, their total rates have to be the same, i.e.,

$$
\begin{aligned}
& 2^{n R_{12}}=2^{n R_{12}^{(1)}+\ldots+n R_{12}^{(N)}}=2^{n R_{12}^{\prime(1)}+\ldots+n R_{12}^{\prime(N)}}, \\
& 2^{n R_{21}}=2^{n R_{21}^{(1)}+\ldots+n R_{21}^{(N)}}=2^{n R_{21}^{\prime(1)}+\ldots+n R_{21}^{\prime(N)}} .
\end{aligned}
$$

An achievable rate region obtained using the re-partitioning in (19)-(20) is given in the following theorem.

Theorem 2: For a two user cooperative OFDMA channel, the rate region described by the closure of the convex hull of all rate pairs $\left(R_{1}, R_{2}\right)$ satisfying

$$
\begin{aligned}
& R_{1}=\sum_{i=1}^{N} R_{10}^{(i)}+R_{12}^{(i)}=\sum_{i=1}^{N} R_{10}^{(i)}+R_{12}^{\prime(i)}, \\
& R_{2}=\sum_{i=1}^{N} R_{20}^{(i)}+R_{21}^{(i)}=\sum_{i=1}^{N} R_{20}^{(i)}+R_{21}^{\prime(i)},
\end{aligned}
$$

with

$$
\begin{aligned}
R_{12}^{(i)} & <C_{12}^{(i)}, \\
R_{21}^{(i)} & <C_{21}^{(i)}, \\
R_{10}^{(i)} & <C_{10}^{(i)}, \\
R_{20}^{(i)} & <C_{20}^{(i)}, \\
R_{10}^{(i)}+R_{20}^{(i)} & <C_{0}^{(i)}, \\
R_{12}^{(i)}+R_{21}^{(i)}+R_{10}^{(i)}+R_{20}^{(i)} & <C_{s}^{(i)},
\end{aligned}
$$

is achievable; where the convex hull is taken over all power allocation policies that satisfy (9), and $C_{12}^{(i)}, C_{21}^{(i)}, C_{10}^{(i)}, C_{20}^{(i)}$, $C_{0}^{(i)}$ and $C_{s}^{(i)}$ are defined in (10)-(15).

Proof: Although the statement of the theorem is very similar to that of Theorem 1, there are now two sets of rates, $R_{k j}^{(i)}$ and $R_{k j}^{\prime(i)}$, arising from the modification we propose in block Markov encoding. The codebook generation, encoding, and decoding policies are obtained by an extension of the approach in [7], to accommodate multiple submessages, as described below.

\section{Codebook Generation:}

The following codebook generation procedure is repeated for each subchannel $i=1, \cdots, N$.

- Generate $2^{n\left(R_{12}^{\prime(i)}+R_{21}^{\prime(i)}\right)}$ length $n$ sequences $u^{(i)}$ with i.i.d. unit Gaussian entries, and assign them to distinct message pairs $\left\{v_{12}^{(i)}, v_{21}^{(i)}\right\} \in\left\{1, \cdots, 2^{n R_{12}^{(i)}}\right\} \times\left\{1, \cdots, 2^{n R_{21}^{\prime(i)}}\right\}$, to form $u^{(i)}\left(v_{12}^{(i)}, v_{21}^{(i)}\right)$.

- For every $u^{(i)}\left(v_{12}^{(i)}, v_{21}^{(i)}\right)$, generate $2^{n R_{12}^{(i)}}$ length $n$ sequences $x_{12}^{(i)}$, and $2^{n R_{10}^{(i)}}$ length $n$ sequences $x_{10}^{(i)}$ from independent unit Gaussian distributions, and 
assign them to distinct $w_{12}^{(i)} \in\left\{1, \cdots, 2^{n R_{12}^{(i)}}\right\}$ and $w_{10}^{(i)} \in\left\{1, \cdots, 2^{n R_{10}^{(i)}}\right\}$ respectively; to form $x_{12}^{(i)}\left(w_{12}^{(i)}, u^{(i)}\left(v_{12}^{(i)}, v_{21}^{(i)}\right)\right)$ and $x_{10}^{(i)}\left(w_{10}^{(i)}, u^{(i)}\left(v_{12}^{(i)}, v_{21}^{(i)}\right)\right)$.

- For every $u^{(i)}\left(v_{12}^{(i)}, v_{21}^{(i)}\right)$, generate $2^{n R_{21}^{(i)}}$ length $n$ sequences $x_{21}^{(i)}$ and $2^{n R_{20}^{(i)}}$ length $n$ sequences $x_{20}^{(i)}$ from independent unit Gaussian distributions, and assign them to distinct $w_{21}^{(i)} \in\left\{1, \cdots, 2^{n R_{21}^{(i)}}\right\}$ and $w_{20}^{(i)} \in\left\{1, \cdots, 2^{n R_{20}^{(i)}}\right\}$ respectively; to form $x_{21}^{(i)}\left(w_{21}^{(i)}, u^{(i)}\left(v_{12}^{(i)}, v_{21}^{(i)}\right)\right)$ and $x_{20}^{(i)}\left(w_{20}^{(i)}, u^{(i)}\left(v_{12}^{(i)}, v_{21}^{(i)}\right)\right)$.

Encoding:

The way block Markov superposition encoding is executed is mostly similar to the case of intra-subchannel cooperative encoding, with the key difference that, in block $b$, the cooperative codeword $U_{k}$ of each user $k$ is now assigned to $\hat{v}_{j k}^{(i)}[b-1]$ and $v_{k j}^{(i)}[b-1]$, which are re-partitionings of the cooperative messages exchanged in the previous block. Then, in each block $b=1, \cdots, B$, appropriate codewords, which correspond to the messages from previous and current block, are selected from the randomly generated codebook above, i.e.,

$$
\begin{aligned}
& X_{k 0}^{(i)}\left(w_{k 0}^{(i)}[b], v_{k j}^{(i)}[b-1], \hat{v}_{j k}^{(i)}[b-1]\right), \\
& X_{k j}^{(i)}\left(w_{k j}^{(i)}[b], v_{k j}^{(i)}[b-1], \hat{v}_{j k}^{(i)}[b-1]\right), \\
& U_{k}^{(i)}\left(v_{k j}^{(i)}[b-1], \hat{v}_{j k}^{(i)}[b-1]\right) .
\end{aligned}
$$

These codewords are superposed using (8), and are subject to the power constraint (9) as in Section III-B.

Note that, in the first block, $b=1$, cooperative messages are set to $\left\{v_{12}^{(i)}[1], v_{21}^{(i)}[1]\right\}=\{1,1\}$; and in the last block, $b=B$, the fresh information is set to $\left\{w_{12}^{(i)}(B), w_{10}^{(i)}(B), w_{21}^{(i)}(B), w_{20}^{(i)}(B)\right\}=\{1,1,1,1\}$, over each subchannel $i=1, \cdots, N$.

\section{Decoding:}

For decoding, each user uses joint typicality check at the end of each block. The receiver on the other hand uses backwards decoding [7] to determine the transmitted messages. That is, in block $B$, the receiver decodes $v_{12}^{(i)}[B-1]$ and $v_{21}^{(i)}[B-1]$ over each subchannel $i$ using joint typicality check, and therefore also knows $w_{12}[B-1]$ and $w_{21}[B-1]$. Then, in block $B-1$, it uses this information to jointly decode $\left\{v_{12}^{(i)}[B-2], w_{10}^{(i)}[B-\right.$ $\left.1], v_{21}^{(i)}[B-2], w_{20}^{(i)}[B-1]\right\}$, based on which it may also deduce $w_{12}[B-2]$ and $w_{21}[B-2]$, and this process continues until the first block.

The decoding operation in an arbitrary block $b$ for each subchannel $i=1, \ldots, N$, is equivalent to finding $\tilde{v}_{12}^{(i)}[b-$ 1], $\tilde{v}_{21}^{(i)}[b-1], \tilde{w}_{10}^{(i)}[b]$ and $\tilde{w}_{20}^{(i)}[b]$ for which

$$
\begin{aligned}
& \left\{y^{(i)}[b], u^{(i)}\left(\tilde{v}_{12}^{(i)}[b-1], \tilde{v}_{21}^{(i)}[b-1]\right),\right. \\
& x_{12}^{(i)}\left(\tilde{w}_{12}^{(i)}[b], u^{(i)}\left(\tilde{v}_{12}^{(i)}[b-1], \tilde{v}_{21}^{(i)}[b-1]\right)\right), \\
& x_{21}^{(i)}\left(\tilde{w}_{21}^{(i)}[b], u^{(i)}\left(\tilde{v}_{12}^{(i)}[b-1], \tilde{v}_{21}^{(i)}[b-1]\right)\right), \\
& x_{1}^{(i)}\left(\tilde{w}_{10}^{(i)}[b], \tilde{w}_{12}^{(i)}[b], u^{(i)}\left(\tilde{v}_{12}^{(i)}[b-1], \tilde{v}_{21}^{(i)}[b-1]\right)\right) \text {, } \\
& \left.x_{2}^{(i)}\left(\tilde{w}_{20}^{(i)}[b], \tilde{w}_{21}^{(i)}[b], u^{(i)}\left(\tilde{v}_{12}^{(i)}[b-1], \tilde{v}_{21}^{(i)}[b-1]\right)\right)\right\}
\end{aligned}
$$

are jointly typical. The estimates of the re-partitioned cooperative messages $\tilde{v}_{k j}^{(i)}[b-1]$ are converted to estimates of the cooperative messages $\tilde{w}_{k j}^{(i)}[b-1]$ using the match-up table available at the users and the receiver.

Using this decoding strategy, and well known properties of jointly typical sequences [7], [15], it can be shown that for $n$ large enough, the average error probability can be made arbitrarily close to zero provided that the rates satisfy the following constraints:

$$
\begin{aligned}
& R_{12}^{(i)}<I\left(X_{12}^{(i)}, Y_{2}^{(i)} \mid X_{2}^{(i)}, U^{(i)}\right), \\
& R_{21}^{(i)}<I\left(X_{21}^{(i)}, Y_{1}^{(i)} \mid X_{1}^{(i)}, U^{(i)}\right), \\
& R_{10}^{(i)}<I\left(X_{1}^{(i)} ; Y^{(i)} \mid X_{2}^{(i)}, X_{12}^{(i)}, U^{(i)}\right), \\
& R_{20}^{(i)}<I\left(X_{2}^{(i)} ; Y^{(i)} \mid X_{1}^{(i)}, X_{21}^{(i)}, U^{(i)}\right), \\
& R_{10}^{(i)}+R_{20}^{(i)}<I\left(X_{1}^{(i)}, X_{2}^{(i)} ; Y^{(i)} \mid X_{12}^{(i)}, X_{21}^{(i)}, U^{(i)}\right), \\
& R_{12}^{(i)}+R_{21}^{(i)}+R_{10}^{(i)}+R_{20}^{(i)}<I\left(X_{1}^{(i)}, X_{2}^{(i)} ; Y^{(i)}\right) .
\end{aligned}
$$

Finally, evaluating (34)-(39) for Gaussian codewords used in encoding, we obtain the desired result.

The improvement in the set of achievable rate pairs $\left(R_{1}, R_{2}\right)$, obtained by inter-subchannel cooperative encoding, becomes more apparent if we restate the achievable rates in Theorem 2 in terms of total rates of the users:

Corollary 2: The achievable rate region given in Theorem 2 is equivalent to the closure of the convex hull of all rate pairs $\left(R_{1}, R_{2}\right)$ satisfying

$$
\begin{aligned}
R_{1} & <\sum_{i} C_{12}^{(i)}+C_{10}^{(i)}, \\
R_{2} & <\sum_{i} C_{21}^{(i)}+C_{20}^{(i)}, \\
R_{1}+R_{2} & <\min \left\{\sum_{i} C_{s}^{(i)}, \sum_{i} C_{12}^{(i)}+C_{21}^{(i)}+C_{0}^{(i)}\right\} .
\end{aligned}
$$

Proof: Constraints (40) and (41) follow trivially from (22)(28). Constraint (42) follows from (22)-(25), (28)-(29) and the fact that $R_{k j}^{(i)}$ and $R_{k j}^{\prime(i)}$ are constrained separately, and need not be equal on a given subchannel $i$; as long as their sum over all subchannels remains the same.

Comparing the achievable rate region (40)-(42) of Corollary 2 with (16)-(18) of Corollary 1, we see that the minimum operations required for the individual rate constraints for each subchannel are removed, and the minimum operation required for the sum rate constraint (18) is taken outside the summation over the subchannels. This way, possible bottlenecks on achievable rates, caused by the per-subchannel constraints, are removed, and the rates obtained by inter-subchannel cooperative encoding are always greater than or equal to those obtained by intra-subchannel cooperation. The rate regions achievable by both strategies will be compared for some sample fading scenarios, in Section IV.

\section{Simulation Results}

In this section we evaluate the achievable rate regions (40)(42) for inter-subchannel cooperative encoding and (16)-(18) for intra-subchannel cooperative encoding, and compare them with the capacity region of a non-cooperative OFDMA system. We assume that, for all three protocols, the users are able to allocate their total power across subchannels and codewords, 


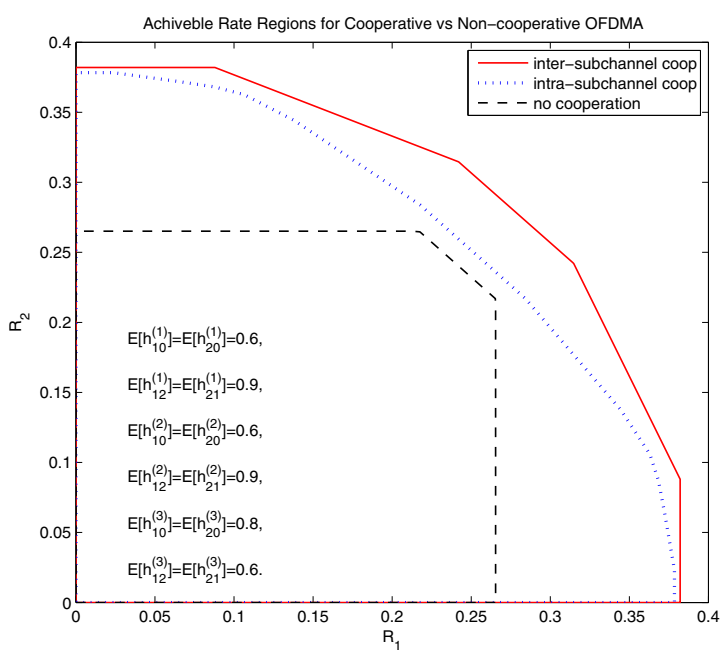

Fig. 1. Achievable rate regions for fading scenario 1.

but power allocation as a function of instantaneous fading states is not considered. Hence, we generate the rate regions using an exhaustive search over all valid distributions of available power over subchannels, as well as over codewords in each subchannel, and taking the convex hull of all achievable rates. The total power of each user and the noise variances are both set to unity. The achievable rate/capacity regions are generated for two separate fading scenarios, which are chosen from independent Rayleigh distributions, the means of which are shown in the respective Figures 1 and 2 along with the results.

In Figure 1, we see that the single user achievable rates for both cooperative strategies are similar, but the gap between the achievable rates of the two policies increases near the sum rate point. This can be explained as follows: the cooperative links on the first two subchannels are better on average, while the direct link is better on the third. Therefore, although the term $C_{s}^{(i)}$ is not very restrictive on the single user rates (16)-(17), it is restrictive for the sum rate (18). Since inter-subchannel cooperative coding can assign powers to cooperative codewords so that it mostly uses the third subchannel to send the cooperative codeword $U$, and the first two for cooperation among users, its advantage near the sum rate point is more pronounced.

In Figure 2, the gap among the two cooperative policies is more apparent, since now the direct link gain of user 1 is worse than user 2 on subchannel 1 , and vice versa on subchannel 2. Therefore, the rate gains achievable in those sub-channels are hindered by the per-subchannel constraints of intra-subchannel cooperation, and in fact around the sum rate point it achieves rates only slightly better than noncooperative OFDMA. Further simulations, the results of which are omitted due to space constraints, show that if the direct links are consistently worse than the cooperative links, the gains from both encoding policies get more and more significant, especially for single user rates.

\section{CONCLUSION}

In this paper we introduced a two user cooperative OFDMA system, and we proposed two encoding strategies: intrasubchannel cooperative encoding and inter-subchannel cooperative encoding, based on block Markov superposition encoding.

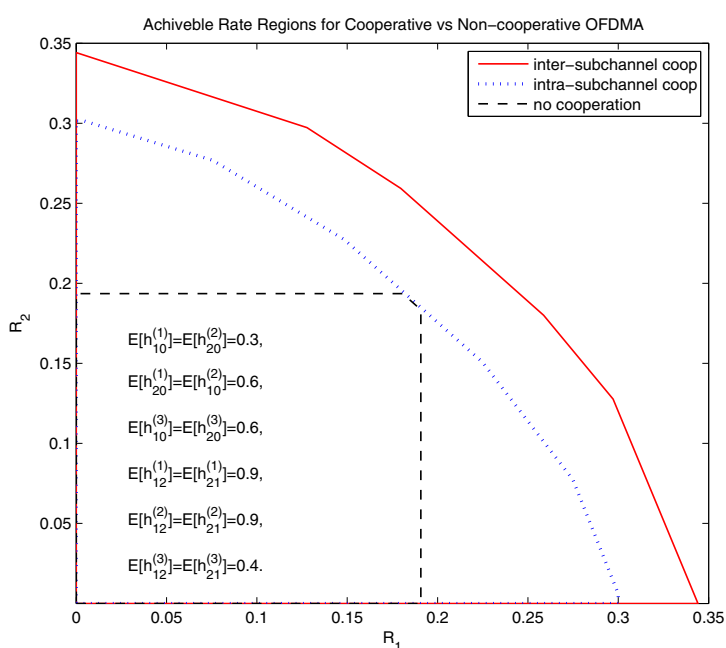

Fig. 2. Achievable rate regions for fading scenario 2 .

We derived rate region expressions for both encoding strategies and showed that re-partitioning and re-encoding of the cooperative messages across subchannels, i.e., inter-subchannel cooperative encoding, is always superior to intra-subchannel cooperative encoding, and provides significant rate gains.

\section{REFERENCES}

[1] J. Jang and K. Lee. Transmit Power Adaptation for Multiuser OFDM Systems. IEEE Journal on Selected Areas in Communications, 21: 171-178, February 2003.

[2] C. Ng and C. Sung. Low Complexity Subcarrier and Power Allocation for Utility Maximization in Uplink OFDMA Systems. IEEE Trans. on Wireless Communications, 7: 1667-1675, May 2008.

[3] I. Wong and B. Evans. Optimal Downlink OFDMA Resource Allocation with Linear Complexity to Maximize Ergodic Rates. IEEE Trans. on Wireless Communications, 7: 962-971, March 2008.

[4] E. C. Van der Meulen. Three-Terminal Communication Channels. Adv. Appl. Prob., 3: 120-154, 1971.

[5] T. Cover and A. Gamal. Capacity Theorems for the Relay Channel. IEEE Trans. on Information Theory, 25: 572-584, Sep 1979.

[6] A. Carleial. Multiple-Access Channels with Different Generalized Feedback Signals. IEEE Trans. on Information Theory, 28(6): 841$850,1982$.

[7] F. M. J. Willems, E. C. van der Meulen and J. P. M. Schalkwijk. An Achievable Rate Region for the Multiple Access Channel with Generalized Feedback. In Proc. Allerton Conference, Monticello, IL, October 1983

[8] A. Sendonaris, E. Erkip and B. Aazhang. User Cooperation Diversity - Part I: System Description. IEEE Trans. on Communications, 51(11): 1927-1938, November 2003.

[9] J. Lin and A. Stefanov. Coded Cooperation for OFDM systems. International Conference on Wireless Networks, Communications and Mobile Computing, 1: 7-10, June 2005.

[10] B. Can, H. Yomo and E. D. Carvalho. Hybrid Forwarding Scheme for Cooperative Relaying in OFDM Based Networks. IEEE International Conference on Communications Proceedings, 45204525, June 2006.

[11] L. Dai, B. Gui and L. Cimini. Selective Relaying in OFDM Multihop Cooperative Networks. IEEE Wireless Communications and Networking Conference, 963-968, March 2007.

[12] L. Weng and R. D. Murch. Cooperation Strategies and Resource Allocations in Multiuser OFDMA Systems. IEEE Trans. on Vehicular Technology, 58(5), June 2009.

[13] W. Shim, Y. Han and S. Kim. Fairness-Aware Resource Allocation in a Cooperative OFDMA Uplink System. IEEE Trans. on Vehicular Technology, 59(2): 932-939, February 2007.

[14] O. Kaya and S. Ulukus. Power Control for Fading Cooperative Multiple Access Channels. IEEE Trans. on Wireless Communications, 6(8): 2915-2923, August 2007.

[15] T. Cover and J. Thomas. Elements of Information Theory. New York: Wiley, 1991. 